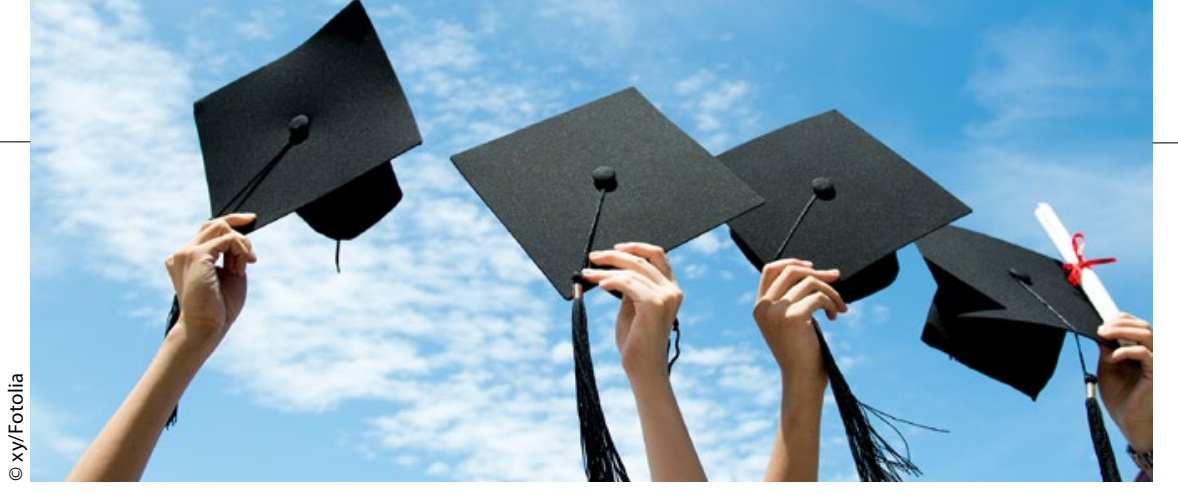

Onlineportale

\title{
Ärzte müssen falsche Titel melden
}

\begin{abstract}
— Ärzte ohne Doktortitel müssen dagegen vorgehen, wenn sie in Internetportalen mit Titel geführt werden, auch wenn sie die Einträge nicht selbst veranlasst haben. Schreiten sie nicht ein, obwohl innen der Fehler bekannt ist, verhalten sich Mediziner pflichtwidrig.

Das hat das Landgericht Hamburg (LG) in einem nicht rechtskräftigen Urteil entschieden (Az.: 312 O 574/15). Die Zentrale zur Bekämpfung unlauteren Wettbewerbs hatte eine Zahnärztin aus Hamburg verklagt, die auf verschiedenen Portalen und auf der Seite eines ärztlichen Vereins mit der Bezeichnung „Dr. med. dent.“ geführt wurde, obwohl sie nie einen Doktortitel erworben hatte. Die Wettbewerbszentrale hatte sie mehrfach darauf hingewiesen und sie aufgefordert, bei den Portalbetreibern für
\end{abstract}

Abhilfe zu sorgen. Die geforderte Unterlassungserklärung gab sie nicht ab. Die Zahnärztin war der Überzeugung, dass sie nicht zu einer Reaktion verpflichtet war. Denn die Wettbewerbszentrale hätte ja selbst gegen die Verwendung des Doktortitels vorgehen können, argumentierte sie vor dem LG.

Das sahen die Richter anders: Sie habe den Titel zwar nicht aktiv verwendet, hafte aber durch "pflichtwidriges Unterlassen“. Nach Einschätzung der Richter konnten die Einträge einen "nicht unerheblichen Werbeeffekt" für die Zahnärztin haben. Die Abmahnung durch die Wettbewerbszentrale hält das LG für berechtigt. Es verurteilte die Zahnärztin unter Androhung eines Ordnungsgeldes in Höhe von $250.000 €$ dazu, die Bezeichnung "Dr. med. dent." nicht zu verwenden, solange sie den Titel nicht erworben hat. (iss)

Fibromyalgiesyndrom

\section{Weitere Leitlinie verfügbar}

— Die beim diesjährigen EULAR-Kongress in London vorgestellte neue britische Leitlinie zur Behandlung bei Fibromyalgie haben sich kaum verändert, allerdings sind sie nun überwiegend Evidenzund nicht mehr nur Eminenz-basiert [Ann Rheum Dis. 2016 Jul 4].

Die Autoren formulierten zwei übergeordnete Prinzipien: Das erste betont, dass eine optimale Behandlung eine frühe Diagnosestellung erfordert. Zudem sollte Fibromyalgie in einem abgestuften Prozess behandelt werden, sagte Professor Gary Mcfarlane vom Institute of Medical Sciences, University of Aberdeen. Als zweites Therapieprinzip nennt die Leitlinie die Verbesserung der gesundheitsbezogenen Lebensqualität, wobei Nutzen und Risiken der Therapiebausteine abgewogen und die nicht pharmakologischen und pharma- kologischen Behandlungen in Kombination und durch ein multidisziplinäres Team erbracht werden sollen. In einer gemeinsamen Entscheidung mit dem Patienten soll die Therapie an dessen individuelle Schmerzintensität, Funktion, Depressionsneigung, Fatigue, Schlafstörungen und Komorbiditäten angepasst werden. Mcfarlane betonte, dass die Therapie gemäß der neuen Leitlinie mit nicht pharmakologischen Interventionen begonnen werden soll. Führen diese Maßnahmen nicht zum Erfolg, sollten individualisiert und an die Patientenbedürfnisse angepasste pharmakologische Therapien zum Einsatz kommen.

(kat)

Zur Fibromyalgie sind bereits Leitlinien der Kanadischen Schmerzgesellschaft, der Deutschen AWMF sowie die Praxisleitlinie Fibromyalgiesyndrom der DGS implementiert.
Neuer Ansatz

\section{Mit Chili-Schärfe gegen Schmerzen}

- Capsaicin kann bekanntlich bei Schmerzen helfen, häufig kommt es jedoch zu Nebenwirkungen wie starkem Brennen. Forscher der Friedrich-Alexander-Universität Erlangen-Nürnberg (FAU) haben nun eine Substanz ausfindig gemacht, die sich ebenso eignen könnte, um starke Schmerzen zu lindern - jedoch weitaus verträglicher ist [Sci Rep. 2016; 6:28621].

Ein Team um Dr. Matthias Engel, Lehrstuhl für Innere Medizin I, und Professor Peter Reeh, Institut für Physiologie und Pathophysiologie der FAU, haben die Substanz Capsazepin, die den Capsaicin-Rezeptor teilweise blockiert, genauer untersucht, heißt es in einer Mitteilung der FAU. Der Stoff hatte in Studien anderer Wissenschaftler Colitis ulcerosa bei Mäusen verhindert. Jedoch musste dafür eine unbekannte Nebenwirkung von Capsazepin verantwortlich sein, denn aus eigenen Untersuchungen wusste Engel, dass der Capsaicinrezeptor am Krankheitsprozess dieser Entzündung gar nicht beteiligt ist. In früheren Arbeiten verhinderte ein synthetischer Hemmstoff des Senföl-Rezeptors TRPA1 die Colitis nicht nur, sondern heilte sie sogar.

Daher vermuteten die Erlanger Forscher, dass Capsazepin eine solche hemmende Nebenwirkung auf TRPA1 haben könnte und erlebten eine Überraschung: Der Wirkstoff hemmte den Rezeptor nicht, sondern aktivierte ihn höchst effektiv. Dies führte dazu, dass er gegen den Reizstoff unempfindlich wird. Obwohl Capsazepin lokal im Darm verabreicht wurde, wurden auch in der Haut kaum noch Neuropeptide ausgeschüttet. Die Autoren folgerten daraus, dass Capsazepin auf dem Blutweg alle Nozizeptoren im Körper wirksam erreichen und vielleicht desensibilisieren kann.

Bei mehrtägiger Gabe von Capsazepin in hoher, aber gut verträglicher Dosis ging die Empfindlichkeit für schmerzhafte chemische und Hitzereize nach und nach im ganzen Körper deutlich zurück, und gleichzeitig wurde die Colitis verhindert. Ein vielversprechendes Ergebnis, das langfristig helfen könnte, hochwirksame Schmerzmittel zu entwickeln, für Krankheiten, bei denen TRPA1 eine wichtige Rolle spielt, teilt die Universität mit.

(eb) 